\title{
DETECTION \& MANAGEMENT OF CONCEPT DRIFT
}

\author{
LEE-ONN MAK, PAUL KRAUSE
}

\author{
Department of Computing, School of Electronics \& Physical Sciences, University of Surrey, UK \\ E-MAIL: 1.mak@surrey.ac.uk,p.krause@surrey.ac.uk
}

\begin{abstract}
:
The ability to correctly detect the location and derive the contextual information where a concept begins to drift is essential in the study of domains with changing context. This paper proposes a Top-down learning method with the incorporation of a learning accuracy mechanism to efficiently detect and manage context changes within a large dataset. With the utilisation of simple search operators to perform convergent search and JBNC with a graphical viewer to derive context information, the identified hidden context are shown with the location of the disjoint points, the contextual attributes that contribute to the concept drift, the graphical output of the true relationships between these attributes and the Boolean characterisation which is the context.
\end{abstract}

\section{Keywords: \\ Concept drift; context; context derivation; Bayesian Network Classifiers}

\section{Introduction}

The ability to correctly detect the location and derive the contextual information where a concept begins to drift is essential in the study of domains with changing context. For example, in the specific case of studying virus behaviour, marketing profiles, medical diseases and more, the correct location of changes can help to produce information that justifies and explains the change.

In the area of detecting and managing context changes, we extend the work of Widmer (METAL) [6] and Harries (SPLICE) [2] and resolve some limitations exhibited by these two systems. METAL(B) uses a Naïve Bayes classifier [1] as the base learner and a statistical method to identify the contextual attributes. Due to the limitations of the Naïve Bayes classifier (the assumption that all attributes are conditionally independent), this classifier can only be used as a black box classifier. The outcome of the learning process is a graph of drifting locations. The characteristics or information at the points of concept drift are not clearly shown.

SPLICE is an offline learner that uses the C4.5 algorithm to perform the initial clustering. The disadvantage of SPLICE is that SPLICE can have poor convergence and has no proof of convergence in some domains. In addition, due to the use of clustering techniques, SPLICE has no notion of overlapping contexts. Further, SPLICE provides no information about the properties of the identified hidden context.

This paper proposes a Top-down learning method with the incorporation of a learning accuracy mechanism to efficiently detect and manage context changes within a large dataset. With the utilization of simple search operators to perform convergent search and JBNC with a graphical viewer to derive context information, the identified hidden context are shown with the location of the disjoint points, the contextual attributes that contribute to the concept drift, the graphical output of the true relationships between these attributes and the Boolean characterisation which is the context.

\section{Learning with Single Context}

A stable concept is a concept that holds true for some period of time. For each collection of data, there exists a hidden context. The group of data instances that contributes to the concept can be considered as a data cluster where the infra similarity is high. Therefore, the self-accuracy should be $100 \%$ accurate if no noise is present in the concept. To handle noise or irrelevant instances, an allowable noise limit is used in a self-accuracy test.

For example, the STAGGER dataset consists of 3 data sets and each dataset has a hidden context. If we join the 3 datasets and learn with JBNC_SFAND [5], the self-accuracy for the total 79 instances is $64.6 \%$ with 28 misclassified instances. However, if the datasets are learned separately, we achieve $100 \%$ for each dataset.

Therefore, this paper is predicated on the principle that the self-accuracy of a dataset that has a single context should be $100 \%$, or slightly less in the presence of noise. If the dataset consists of multiple contexts, the learning algorithm will be confused, with a corresponding reduction in the learning accuracy. 


\section{Context Derivation}

To illustrate the method in deriving context, we give a brief description of the techniques. For full details of the example used, please refer to [4].

JBNC [3], a Java toolkit for training, testing and applying Bayesian Network classifiers, was designed and written by Sacha [5].

In this paper, JBNC is selected as the underlying learner as it overcomes the limitation of Naïve Bayes classifier [4], in producing a precise network structure, which is useful in formulating context. The JBNC SAND operator discards attributes that are not determined to be dependent on the class variable before applying the AUGMENTER operator. The graphical outcome is a set of relevant attributes, the arcs that linked the attributes, and the attributes' value with reference to the probability tables.

In this paper, context is represented by a Boolean characterisation, using Boolean operators to represent the conjunctive or disjunctive nature of the learnt representation. There can be "AND", "OR" or both conditions in representing the underlying context of the dataset. From the graphical output of the learnt Bayesian network structures, the identification of the conjunction "AND" and disjunction "OR" relationship is as follows:



(a)

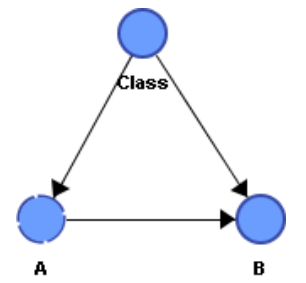

(b)
Figure 1. (a) The "AND" relationship between attributes,

(b) The "OR" relationship between attributes

In Figure 1a, the Bayesian network can model a concept represented as "A AND B". From the diagram, there are arcs connected from the class node to each attribute node. To portray the "AND" relationship, there is no direct arc from attribute A to attribute B or vice versa. This means that there is no dependency between the attribute nodes of $\mathrm{A}$ and $\mathrm{B}$. This captures the following cases: if $C$ is true, then $A$ is true; if $C$ is true, then $B$ is true. That is, if $\mathrm{C}$ is true, then both $\mathrm{A}$ and $\mathrm{B}$ are true.

However, if the preferred representation of a concept is "A OR B" (Figure 1b), then we must place a direct dependency between the attribute nodes of A and B. This is capturing the following cases: if $\mathrm{A}$ is false, then $\mathrm{B}$ must be true; if $B$ is false, then A must be true.

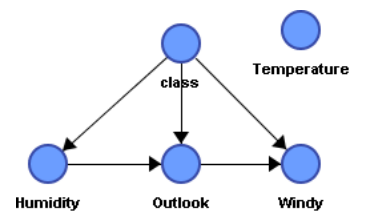

Figure 2. The learnt network structure of Weather dataset

Figure 2 shows the learnt network structure of the Weather datasets. From the figure, there are arcs that link Humidity to Outlook and Outlook to Windy which symbolises the "OR" relationship. There is no direct link between Humidity and Windy which symbolises the "AND" relationship. From the probability tables in [4], the derived context represented by Figure 2 is (Humidity = Normal AND Windy $=$ False) OR Outlook $=$ Overcast.

\section{Brief Description of Process Flow}

To detect concept drift and identify context information, we present an efficient method which overcomes the limitation of SPLICE and METAL.

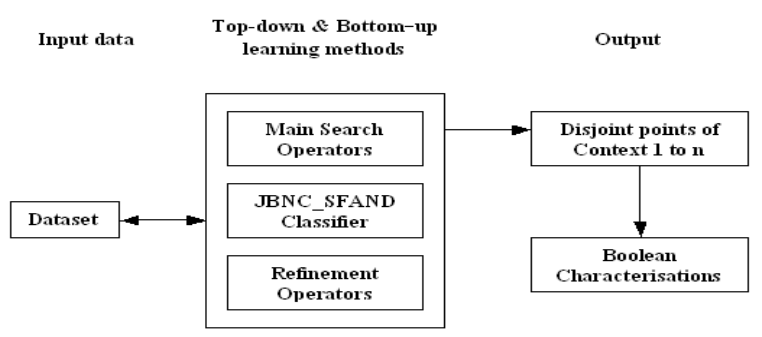

Figure 3. The process of detecting concept drift

Figure 3 illustrates the general process of detecting concept drift over time. The datasets that feed to the learning system are collected over time and may contain hidden changes of context. The learning system seeks to detect the "disjoint" points within the dataset where the concept begins to drift. This method uses the JBNC_SFAND classifier, the main search operators and the refinement operators to detect the actual disjoint points within the dataset. The outcomes of the learning are the clusters of data instances, which are delimited by the location of the disjoint points, and their Boolean characterisations which represent the context.

There are 2 learning methods created in our research work in [4]. For this paper, we focus the presentation on the "Top-down" learning method. 


\section{Search Operators}

The main task of the learning algorithm is to efficiently search for the disjoint point where the concept begins to drift. This section proposes 3 operators to perform the main and refinement searches.

As the main search operator, the rate reduction operator seeks to achieve quick convergence to the approximate disjoint point within a large dataset. The operator performs an instance reduction based on a user-specified rate. The reduction rate ranges from 0 to $100 \%$. For example, if there are 100 instances in the dataset, after the rate reduction of $50 \%$, the leftover instances will be 1 to 50 and the instances from 51 to 100 are removed.

The misclassified removal operator searches the approximate disjoint point by removing the number of misclassified instances from the present instances that produce the network structure. This operator activates after the main operator has completed the initial searching. The operator is used to remove the outliers or irrelevant instances that do not belong to the present group of instances that formulate that concept. The condition to activate this operator is that the present stage of learning has achieved accuracy below the allowable noise limit but the previous stage had achieved error rate greater than the allowable noise limit.

The step operator refines the result by making a step forward search from the approximate disjoint point. This operator is activated after the main operator or the misclassified removal operator has completed the convergent search. This operator is used to perform a single step or user-specified step to reach the actual disjoint point.

\section{Top-down Method}

The Top-down learning method uses the various operators mentioned above to search for the actual disjoint points within a dataset according to a user-specified reduction rate. By "Top-down" we mean that the whole dataset is involved in the initial learning and the subsequent reduction of instances is done from the end of the dataset. Table 1 shows the learning algorithm.

From Figure 4, the search begins with the total instances $n$. At this step, the JBNC SFAND classifier is called to learn the dataset and to perform a self-accuracy test. If the misclassification rate (error) is greater than the allowable limit (allow), the algorithm engages the rate reduction operator to perform instance reduction operation. At each iteration, the dataset is reduced by $r \%$ where $r$ is the user-specified reduction rate. For example, the dataset after reduction at iteration 1 is $r \times n$ instances. At the next iteration, the dataset is further reduced to $r \times \mathrm{x} n$ instances.

Table 1. The Top-down Learning Algorithm

Input:

- Dataset with instances n

- Allowable error rate, allow

- User-specified rate reduction $r$

- $\quad$ Step increment 1

Algorithm:

Stage 1: Convergent Search

- Begins with dataset of instances $n$

- Use JBNC_SFAND to classify the dataset and perform self-accuracy test

- If error $>$ allow, call rate reduction operator

- If error $<$ allow, proceed to Stage 2

Stage 2: Refinement Search

- Perform check 1

○ Last iteration step $\mathrm{m}$, error rate $<=$ allow

- Previous iteration step $\mathrm{m}-1$, error $>$ allow

- If check 1 is TRUE, call misclassified removal operator

- If error $<$ allow, proceed to Stage 3

Stage 3: Fine-tuning Search

- $\quad$ Perform check 2

- Last iteration step $\mathrm{m}$, error $<$ allow

- If check 2 is TRUE, call step operator

- If error > allow, stop the search

- Output "Context $x$ is found at instances y"

Stage 4: Data Removal

- Remove instances $y$ from the beginning of instances $n$

- If $\mathrm{n}-\mathrm{y}>0$, goto Stage 1

- If $\mathrm{n}-\mathrm{y}=0$, stop the whole process

Output:

- Location of stable concepts' disjoint points

- Graphical output of learnt Bayesian network structures

- Boolean characterization of the hidden context

For the refinement search, in Figure 4, the rate reduction operator, with much iteration, reaches the approximate disjoint point by performing instances reduction. However, the actual disjoint point is located somewhere after the approximate disjoint point. The misclassified removal operator is used at this point to 
further converge to the right location. The misclassified removal operator attempts to reach the actual disjoint point by removing the number of misclassified instances from the dataset. This process brings the location point closer to the actual disjoint point but not the actual point. The iteration ceases when the error rate goes below the allowable noise limit.

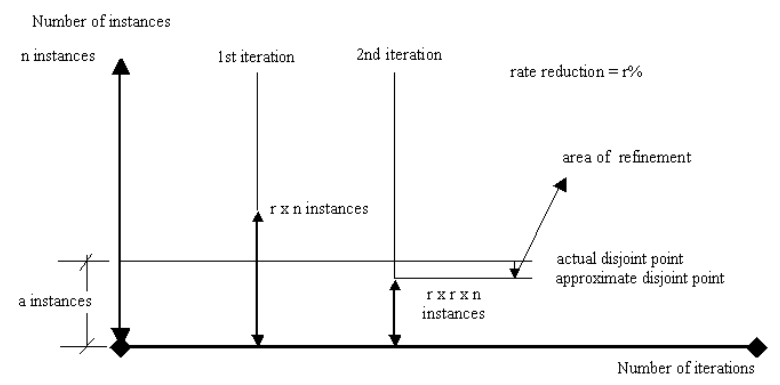

Figure 4. The Top-down searching process

To "fine-tune" to the actual disjoint point, the step operator is employed to search upwardly in a step-by-step manner to reach the actual disjoint point. At each iteration, the dataset is increased by 1 instance or a user-specified number of instances which is greater than 1. Once the error rate exceeds the allowable noise limit, iteration ceases and the actual disjoint point is the number of instances belonging to the previous step.

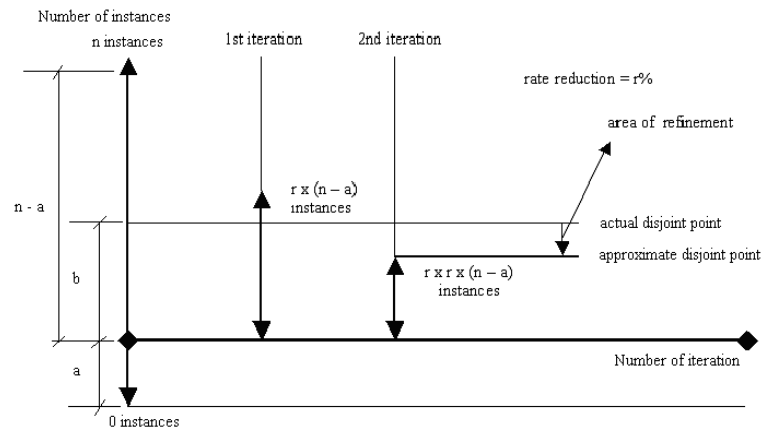

Figure 5. The searching for next context with the removal of $a$ instances

With the final search performed by the step operator, the actual disjoint point for Context 1 is found. To begin the next context search, the pointer line starts to move up to instances $a$ as shown in Figure 5 where $a$ is the $1^{\text {st }}$ disjoint point. This means that the instances that belong to Context 1 are removed from the actual dataset. The next search is done with instances $n$ - $a$. The algorithm performs a test to ensure that the number of instances for the next search is greater than zero. If the test is true, the process repeats stage 1 to 3 . The search iteration continues until the actual disjoint point is found with $\mathrm{b}$ instances. The search process continues as long as the number of instances is greater than zero. If the number of instances equals to zero, the search process ceases.

To ensure good accuracy in locating the right disjoint points, the parameters used in the learning have to be handled efficiently.

The allowable noise limit is used to control the level of noise present in the dataset. No one knows a priori how much noise is present in the dataset that is being tested. Usually, the dataset is tested a few times with the learning method to investigate the noise level. The location of the disjoint points varies according to the allowable noise limit used in the search. After each round of testing, the allowable noise limit needs to be adjusted accordingly.

The reduction rate specified by the user has to be realistic. The recommended values for the reduction rate are from $20 \%$ to $50 \%$. The smaller the reduction, the more accurately and confidently the approximate disjoint points are located.

Finally, the step increment for the refinement search is recommended to be 1 . If the increment is too large, the actual disjoint point cannot be found. With an increase of 1 instance, the actual disjoint point can be located easily.

\section{Example with STAGGER dataset}

In this section, the STAGGER dataset is used to verify the Top-down learning method. This simple dataset consists of 79 instances and 3 hidden contexts where 26 instances belong to context 1, 27 instances belong to context 2 and 26 instances belong to context 3 .

Searching method: Top-down

JBNC: SFAND, LC, STAGGER Dataset

Allowable noise tolerance $=0$

Reduction rate $=50 \%$

*NG means "No Good"

Search No 1.

Total instances $=79$

\begin{tabular}{|l|l|l|l|l|}
\hline $\begin{array}{l}\text { Ste } \\
p\end{array}$ & Action & Inst & $\begin{array}{l}\text { Accuracy/ } \\
\text { Misclassified }\end{array}$ & Remk \\
\hline 1 & & 79 & $65 \% / 28$ & NG \\
\hline 2 & Reduce $50 \%$ & 40 & $82.5 \% / 7$ & NG \\
\hline 3 & Reduce $50 \%$ & 20 & $100 \% / 0$ & OK \\
\hline
\end{tabular}


Refinement Search:

\begin{tabular}{|l|l|l|l|l|}
\hline 4 & $\begin{array}{l}\text { Remove 7 inst } \\
\text { from 40 inst in } \\
\text { step 2 }\end{array}$ & 33 & $84.9 \% / 5$ & NG \\
\hline 5 & $\begin{array}{l}\text { Remove 5 inst } \\
\text { from 33 inst in } \\
\text { step 4 }\end{array}$ & 28 & $92.9 \% / 2$ & NG \\
\hline 6 & $\begin{array}{l}\text { Remove 2 inst } \\
\text { from 28 inst in } \\
\text { step 5 }\end{array}$ & 26 & $100 \% / 0$ & OK \\
\hline
\end{tabular}

Fine-Tuning Search:

\begin{tabular}{|l|l|l|l|l|}
\hline 7 & $\begin{array}{l}\text { Add } 1 \text { inst to } \\
26 \text { inst in step } \\
6\end{array}$ & 27 & $96.3 \% / 1$ & NG \\
\hline
\end{tabular}

Step 6: misclassified rate is $0<=$ allowable

*Context 1 found with $<26>$ instances at $<100 \%>$ accuracy*

**Remove 26 instances from dataset**

Search No 2.

Total instances $=79-26=53$

\begin{tabular}{|l|l|l|l|l|}
\hline $\begin{array}{l}\text { Ste } \\
p\end{array}$ & Action & Inst & $\begin{array}{l}\text { Accuracy/ } \\
\text { Misclassified }\end{array}$ & Remk \\
\hline 1 & & 53 & $56.6 \% / 23$ & NG \\
\hline 2 & Reduce $50 \%$ & 27 & $100 \% / 0$ & OK \\
\hline
\end{tabular}

Refinement Search:

\begin{tabular}{|l|l|l|l|l|}
\hline 3 & $\begin{array}{l}\text { Remove 23 } \\
\text { inst from 53 } \\
\text { inst in step 1 }\end{array}$ & 30 & $93.3 \% / 2$ & NG \\
\hline
\end{tabular}

Fine-Tuning Search:

\begin{tabular}{|l|l|l|l|l|}
\hline 4 & $\begin{array}{l}\text { Add 1 inst to } \\
27 \text { inst in step } \\
2\end{array}$ & 28 & $96.4 \% / 1$ & NG \\
\hline
\end{tabular}

Step 2: misclassified rate is $0<=$ allowable

*Context 2 found with $<27>$ instances at $<100 \%>$ accuracy*

**Remove 27 instances from dataset**

Search No 3.

Total instances $=53-27=26$

\begin{tabular}{|l|l|l|l|l|}
\hline $\begin{array}{l}\text { Ste } \\
p\end{array}$ & Action & Inst & $\begin{array}{l}\text { Accuracy/ } \\
\text { Misclassified }\end{array}$ & Remk \\
\hline 1 & & 26 & $100 \% / 0$ & ok \\
\hline
\end{tabular}

Step 1: misclassified rate is $0<=$ allowable

*Context 3 found with $<26>$ instances with $<100 \%>$ accuracy*

$* *$ Remove 26 instances from dataset**

$* * *$ Total Instances $=0 * * *$

$* * * *$ End of contexts search $* * * *$

Network Structures Output \& Derived Context

Concept drifts from instances 1 to 26, 27 to 53, 54 to 79 with context information as:

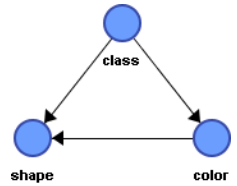

Context 1: ->

color $=$ green or shape $=$ circular

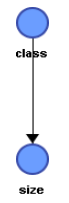

Context 2: ->

size $=$ medium or size $=$ large



Context 3:

$($ size $=$ small $)$ or (color $=$ red and shape $=$ square)

\section{Managing context Accuracy}

In order to manage context accuracy in learning real-life and noisy datasets, the irregularities occurring during learning have to be handled efficiently. The Top-down learning method might experience that the main operator fails to converge to the approximate disjoint point with the possible scenarios:

- The error rate remains above the allowable noise limit throughout the entire search.

- The search operators have missed the approximate disjoint point.

Under these situations, the learning algorithm will behave as if an irregularity has occurred and the iteration ceases. Based on the error rate collected at each learning step, the algorithm engages the interpolation operator to perform a further search for the approximate disjoint point.

The interpolation operator is a special operator that is only activated when irregularities occur during the search. This operator uses the equation: $(x+y) / 2$ where $x$ and $y$ are the number of instances at step $n$ and $n-1$. Both error rates at these steps are greater than the allowable noise limit. The iteration continues as long as the error rate exceeds the allowable noise limit.

\section{Further Experiments}

Next, to verify the performance of the proposed learning method in detecting concept drift in a real-life environment; we apply them to the Music Chord datasets. In [4], the learning method had successfully applied to both Music and Vowel datasets. For further results and explanation of both processes, please refer to [4].

Widmer [6] uses the Music dataset to predict online what chord should accompany the next note in a given melody. The task is to correctly predict one of three classes: tonic harmony, dominant or other. The data used are the melodies of Franz Schubert's German Mass; a collection of 8 songs of varying length. After putting all the songs together for the context search, there are 553 melody notes 
in total.

First, the dataset is tested if only one context lies within the entire dataset. If there is more than one context present in the dataset, the dataset is tested by parts by the rate reduction operator in a Top-down manner. With a $50 \%$ rate reduction, the 553 instances used in search 1 was tested, and then followed by $227,139,70$ and 35 until the error rate is within the allowable noise limit. Search $1,2,4,5,6$, 7 and 8 , with the exception of 3 , have a straightforward error convergent.

After the rate reduction operator reaches the initial disjoint point, the misclassified removal operator is used to bring the location closer to the actual disjoint point. The misclassified removal operator removes the misclassified instances from the present instances. Search 1, 3, 4 and 7 have a straightforward processing. Search 2 and 6 need more iteration before reaching the approximate disjoint point. Hence, the misclassified removal operator is a powerful tool to remove redundant instances and convergence is assured.

The Top-down method handles the irregularity that occurred during the search efficiently by engaging the interpolation operator. In search 3 , after much iteration performed by the rate reduction operator, the approximate disjoint point cannot be obtained as the error rate begins to diverge after the initial convergence. The main search by the rate reduction operator has overlooked the initial disjoint point. By employing the interpolation operator, remedial work is performed and the tentative point of convergence is found. Later, with the misclassified removal operator, the approximate disjoint point is found.

Table 2. The comparison of the searched locations closeness to the actual results

\begin{tabular}{|c|c|c|c|}
\hline Chord & Actual & Searched & Diff \\
\hline 1 & 63 & 62 & 1 \\
\hline 2 & 133 & 139 & 6 \\
\hline 3 & 206 & 212 & 6 \\
\hline 4 & 248 & 254 & 6 \\
\hline 5 & 293 & 329 & 36 \\
\hline 6 & 379 & 368 & -11 \\
\hline 7 & 440 & 456 & 16 \\
\hline 8 & 553 & 553 & 0 \\
\hline
\end{tabular}

From the results produce from search 1 to 8 , we can observe that the search algorithms are simple and convergence can be achieved easily. With the incorporation of learning accuracy mechanisms, the irregularities can be overcome easily.

Table 2 shows the result of the search for disjoint points as compared to the actual locations.

\section{Conclusions \& Discussions}

With the development and integration of algorithms, this paper proposes a rich learning package to identify context and detect the locations where the concepts begin to drift. So far, the learning method produced encouraging results with the Weather and Music datasets.

As compared with the METAL learning system, the JBNC_SFAND classifier outperforms the Naïve Bayes classifier in producing a precise and correct network structure where the context information can be identified easily. With the use of the nodes discarding facility, the JBNC provides the relevant or contextual attributes after learning without using any external statistical method as in METAL.

The learning method has also resolved some of the limitations demonstrated by the SPLICE learning system. SPLICE suffers from convergence problem due to the use of clustering techniques. The Top-down method efficiently converged to the right disjoint point with the use of simple operators. The simple operators had also been demonstrated to be effective on the real-world Music dataset. With the use of allowable noise limits, the Top-down method addressed the issue of overlapping instances. The locations that separated different Music Chord contexts are close to the actual results (Table 2). With the use of JBNC and the graphical viewer, the properties of the identified hidden context are shown with the location of the disjoint point, the contextual attributes that contribute to the concept drift, the graphical output of the true relationships between these attributes and the Boolean characterisation which is the context.

\section{References}

[1] R. O. Duda and P. E. Hart, Pattern Classification and Scene Analysis, John Wiley \& Sons, New York, 1973.

[2] M. B. Harries, C. Sammut and K. Horn, "Extracting Hidden Context", Machine Learning, Vol 32, pp. 101-126, 1998.

[3] JBNC, Bayesian Network Classifier Toolbox, http://jbnc.sourceforge.net.

[4] Lee-Onn Mak, "Identification and Management of Context with A Bayesian Network Classifier", Doctoral dissertation, Department of Computing, School of Electronics \& Physical Science, University of Surrey, July, 2004.

[5] J. P. Sacha, L. Goodenday, K. J. Cios, "Bayesian Learning For Cardiac SPECT Image Interpretation", Artificial Intelligence in Medicine, Vol 26, pp. 109-143, 2002.

[6] Gerhard Widmer, "Tracking Context Changes through Meta-Learning", Machine Learning, Vol 27, No. 3, pp. 259-286, 1997. 